\title{
Phage-display derived single-chain fragment variable (scFv) antibodies recognizing conformational epitopes of Escherichia coli heat-labile enterotoxin B-subunit
}

\author{
Wen Yuan Chung ${ }^{1,7}$, Markus Sack ${ }^{2}$, Rachel Carter $^{3}$, Holger Spiegel ${ }^{4}$, Rainer Fischer ${ }^{2,4}$, Timothy R. \\ Hirst $^{5}$, Neil A. Williams ${ }^{6}$, Roger F.L. James ${ }^{7^{*}}$ \\ ${ }^{1}$ Royal College of Surgeons, Department of Surgery, Connolly Hospital, Blanchardstown, D15, Ireland, \\ ${ }^{2}$ Department of Molecular Biotechnology, RWTH Aachen University, 52074 Aachen, Germany, \\ ${ }^{3}$ CRUK Clinical Centre, Southampton, S016 6YD, UK, \\ ${ }^{4}$ Fraunhofer IME, Institute for Molecular Biology and Applied Ecology, 52074 Aachen, Germany, \\ ${ }^{5}$ Cellular Microbiology, John Curtin School of Medical Research, Australian National University, \\ Canberra, ACT 0200, Australia, \\ ${ }^{6}$ Department of Cell and Molecular Medicine, University of Bristol, Bristol BS8 1TD and \\ ${ }^{7}$ Department of Infection, Immunity and Inflammation, University of Leicester, Leicester, LE1 9HN, UK. \\ *Corresponding author: Dr. Roger F.L. James, Department of Infection, Immunity and Inflammation, \\ University of Leicester, Maurice Shock Building, University Road, Leicester LE1 9HN, UK. Phone: 44 \\ (0)116 2231406 44(0)116 2525033; Fax: 0116 2525035; Email:rj1@le.ac.uk
}

Abbreviations: E.coli, Escherichia coli; scFv, single-chain Fv antibody fragment; ELISA, enzyme-linked immunosorbent assay; $\mathrm{G}_{\mathrm{M} 1}$, monoganglioside- $\mathrm{G}_{\mathrm{M} 1}$ (Gal $\beta-3 \mathrm{GalNac} \beta 1$-(neu5Ac $\left.\alpha 2-3\right)-4 \mathrm{Gal} \beta 1-4 \mathrm{Glc} \beta 1$-cer); Etx, heatlabile enterotoxin; $\mathrm{EtxB}_{5}$, heat-labile enterotoxin $\mathrm{B}$ pentamer; $\mathrm{EtxB}_{1}$, heat-labile enterotoxin $\mathrm{B}$ subunit monomer; Ctx, cholera toxin; mAb, monoclonal antibody; SDS-PAGE, sodium dodecylsulphate polyacrylamide gel electrophoresis; CDR, complementary determining region; $\mathrm{V}_{\mathrm{H}}$, heavy chain of an antibody; $\mathrm{V}_{\mathrm{L}}$, light chain of an antibody; DTT, dithiotreitol; PBS, phosphate buffer saline; MBP, myelin basic protein.

Previously we have described studies on in vitro pentamer assembly of Escherichia $\underline{\text { coli }}$ (E. coli) derived heat-labile enterotoxin B subunit $(\mathrm{EtxB})$ using conventional monoclonal antibodies (Amin $\underline{e t} \underline{a l}$.

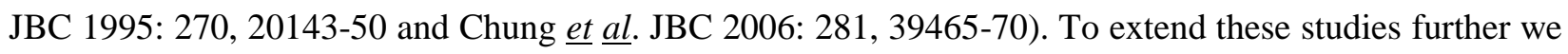
have used phage-display to select single-chain Fragment variable ( $\mathrm{scFv}$ ) antibodies against different forms of the B-subunit. Two clones exhibiting strong and differential binding were chosen for detailed characterization. A comprehensive sequence analysis was performed to assign the framework and complementary-determining regions and a nonsense mutation present in one of these (scFv-B1.3.9) was 
corrected. Binding analysis showed that scFv-B1.3.9 bound in ELISA to both heat-denatured monomeric B-subunits $\left(\mathrm{EtxB}_{1}\right)$ and also displayed cross-reactivity towards pentameric EtxB (EtxB $\mathrm{B}_{5}$, although there was no reactivity towards monoganglioside $\left(\mathrm{G}_{\mathrm{M} 1}\right)$ captured $\mathrm{EtxB}_{5}$. Another antibody (scFvB5.2.14) had a different reactivity profile and, in ELISA, bound only to $\mathrm{EtxB}_{5}$ but not to $\mathrm{EtxB}_{1}$ or to $\mathrm{EtxB}_{5}$ captured via $\mathrm{G}_{\mathrm{M} 1}$. Surprisingly, in competition experiments, the assembled pentameric B-subunit inhibited binding of scFv-B5.2.14 to immobilized $\mathrm{EtxB}_{5}$ only weakly, whereas reduced, but not oxidized, monomeric EtxB $\mathrm{B}_{1}$ was an efficient competitor. These results clearly demonstrate that B1.3.9 and B5.2.14 have different specificities for cryptic epitopes not accessible in the fully assembled $\mathrm{G}_{\mathrm{M} 1}$ bound pentameric form of EtxB.

Taken together our results show that we were able to successfully isolate and characterize recombinant scFvs that differentially recognize diverse denatured forms or assembly intermediates of the heat-labile enterotoxin B subunit of $\underline{E} \underline{\text { coli }}$.

\section{KEYWORDS}

EtxB, epitope, protein assembly, differential recognition, phage display, recombinant antibodies, scFvs

Heat-labile enterotoxin (Etx) produced by certain strains of $\underline{E}$ coli is a major virulence factor related to cholera toxin $(\mathrm{Ctx})$ produced by Vibrio cholera and results in diarrhoeal disease in humans

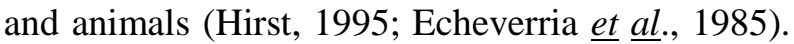
Heat-labile enterotoxin consists of 5 identical Bsubunits $(\mathrm{Mr}=12,000)$, which $\underline{\text { in }} \underline{\text { vivo }}$ naturally assemble into a stable pentameric structure with an A-subunit $(\mathrm{Mr}=28,000)$, which has ADP-ribosyl transferase activity (Holmgren $\underline{\text { et }} \underline{\text { al., }}$ 1975; Fishman $\underline{\text { et }} \underline{a l} .$, 1978; Fishman $\underline{\text { et }} \underline{\text { al., }}$ 1980). The Bsubunit pentamer of Etx $\left(\mathrm{EtxB}_{5}\right)$ specifically recognizes, and has a high affinity for, the oligosaccharide portion of $\mathrm{G}_{\mathrm{M} 1}$ on the surface of intestinal cells and acts to deliver the A-subunit 
where it catalyses ADP-ribosylation of the signal transduction protein $\mathrm{G}_{\mathrm{s}}-\alpha$. By effectively blocking deactivation of $\mathrm{G}_{\mathrm{s}}-\alpha$ intracellular levels of cAMP rise, leading to fluid and $\mathrm{Cl}^{-}$loss from the cells and thus severe diarrhoea (Kuziemko $\underline{e t} \underline{a l} ., 1996$; Turnbull $\underline{\text { et }} \underline{\text { al., 2004). }}$.

In addition to their roles as mediators of diarrhoeal disease, Etx and Ctx have long been known to be potent mucosal immunogens and, when co-administered with other antigens, can give rise to strong tolerogenic responses (Weiner, 1997). In addition, it has been shown that recombinant preparations of $\mathrm{EtxB}_{5}$ affect immunoregulatory processes and lead to the suppression of inflammatory responses (Shevach, 2000). The enterotoxins Etx and Ctx are highly related showing $80 \%$ sequence identity and stimulate strong mucosal immunity both to themselves and to co-administered antigens (Yamamoto $\underline{\text { et }} \underline{\text { al., }}$ 1987; Wilson $\underline{\text { et }} \underline{\text { al., }}$ 1991; Takahashi $\underline{\text { et }} \underline{\text { al., }}$ 1996). The ability of EtxB and CtxB to enhance $\mathrm{T}_{\mathrm{H} 2}$ and suppress $\mathrm{T}_{\mathrm{H} 1}$ inflammatory responses in an antigen-specific manner has been subject to intensive research, particularly in relation to the treatment of autoimmune disease (Williams $\underline{\text { et }} \underline{\text { al., }}$ 1999; Sobel $\underline{e t} \underline{a l} ., 1998 ;$ Luross $\underline{e t} \underline{a l} ., 2002)$. It was initially thought that the adjuvant properties of EtxB were dependent on receptor binding activity as a nonbinding point mutant of EtxB (G33D), lost all immunomodulatory activity. However, the CtxB mutant, CtxB (H57A) was shown to retain the ability to bind to $G_{M 1}$ but was unable to act as an immunomodulator (Truitt $\underline{\text { et }} \underline{\text { al. }} ., 1998$; Williams $\underline{e t}$ al., 1999; Aman $\underline{\text { et }} \underline{\text { al. }} ., 2001$; Fraser $\underline{\text { et }} \underline{\text { al. }} ., 2003)$. It is now theorized that structural alterations in $\mathrm{CtxB}$ (H57A), while not preventing binding to $\mathrm{G}_{\mathrm{M} 1}$, may preclude interactions with additional co-receptors required for signaling (Fraser $\underline{e t} \underline{\text { al., }}$ 2003). Therefore, ganglioside recognition alone, while necessary to achieve high-affinity binding to cell surfaces, may not be sufficient to mediate the immunomodulatory effects of the B subunits.

Previously we reported on the generation and characterization of mAbs specific for $\mathrm{EtxB}_{5}$ (LDS16) and $\mathrm{EtxB}_{1}$ (LDS47) and showed that 
these could be used to study pentamer assembly $\underline{i n}$ vitro and this has led us to propose a model describing the processes of pentamer assembly (Lesieur, 2002). However, investigating in vivo toxin assembly and transport to the periplasmic space using mAb probes is not possible using conventional monoclonal antibodies. In this study we have selected scFvs that specifically react with intermediate forms of EtxB with the potential to be used to study pentamer assembly $\underline{i n} \underline{v i v o}$.

\section{EXPERIMENTAL PROCEDURES}

Phage display library and phage rescue Antibodies against different forms of EtxB were selected from the semi-synthetic scFv phage display library (a generous gift from Dr. Andrew Griffiths, MRC, Cambridge, UK). The $\mathrm{V}_{\mathrm{H}}$ and $\mathrm{V}_{\mathrm{L}}$ regions were re-cloned from the lox library vectors into the phagemid pHEN2 (Griffiths $\underline{e t} \underline{a l} .$, 1994) where the recombinant $\mathrm{scFv}$ genes are flanked by

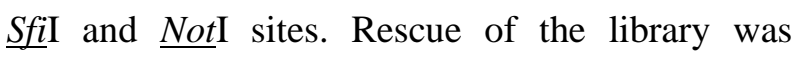
performed as recommended (Griffiths $\underline{e t} \underline{\text { al. }}$, 1994).
Briefly, $10^{10} \mathrm{CFU}$ of the Griffin.1 library in bacterial strain TG1 were inoculated into $2 \mathrm{xTY}$ medium containing $100 \mu \mathrm{g} / \mathrm{ml}$ ampicillin and $1 \%$ glucose. Cells were incubated at $37^{\circ} \mathrm{C}$ until the $\mathrm{OD}_{600}$ reached 0.5 . Then $25 \mathrm{ml}$ of this culture was infected with $10^{10} \mathrm{PFU}$ of VCS-M13 helper phage and incubated for $30 \mathrm{~min}$ at $37^{\circ} \mathrm{C}$. The infected cells were collected by centrifugation for $10 \mathrm{~min}$ at $2,500 \mathrm{~g}$ and resuspended in $300 \mathrm{ml}$ of $2 \mathrm{xTY}$ containing $100 \mu \mathrm{g} / \mathrm{ml}$ ampicillin and $25 \mu \mathrm{g} / \mathrm{ml}$ kanamycin. Subsequently, the bacteria were cultured overnight at $37^{\circ} \mathrm{C}$ with slow rotation (200 rpm). After centrifugation for $30 \mathrm{~min}$ at 2,500 $g$ the supernatant was collected and phages were precipitated twice with $20 \%$ polyethylene glycol 6,000 and $2.5 \mathrm{M} \mathrm{NaCl}$. The phage pellet was resuspended in PBS to a final titer of $10^{12-13}$ PFU/ml.

$$
\text { Biopanning - Nunc immunotubes }
$$
(maxisorp®) were coated overnight with $4 \mathrm{ml}$ of monomeric and pentameric $\operatorname{EtxB}(10 \mu \mathrm{g} / \mathrm{ml})$ at room temperature in $50 \mathrm{mM}$ carbonate buffer, $\mathrm{pH}$ 9.6. After washing three times with PBS the tubes 
were blocked with $2 \%$ milk in PBS and incubated at $37^{\circ} \mathrm{C}$ for $2 \mathrm{~h}$. Phages $\left(10^{12} \mathrm{PFU}\right)$ were diluted into $4 \mathrm{ml}$ of $2 \%$ dried milk powder in PBS and were incubated for $30 \mathrm{~min}$ at room temperature with slight agitation followed by $90 \mathrm{~min}$ at room temperature without agitation. The tubes were thoroughly washed ten times with PBS containing 0.1\% Tween-20, and then ten times with PBS. Phages were eluted by $10 \mathrm{~min}$ incubation with $1 \mathrm{ml}$ of $100 \mathrm{mM}$ triethylamine under slight agitation. The eluted phages were then immediately neutralized with $0.2 \mathrm{ml}$ of $1 \mathrm{M}$ Tris $\mathrm{pH}$ 7.4. The immuno-tubes were rinsed with $0.2 \mathrm{ml}$ $1 \mathrm{M}$ Tris $\mathrm{pH} 7.4$ to neutralize the remaining phages and $0.75 \mathrm{ml}$ of the eluted phages were added to $9.25 \mathrm{ml}$ of an exponentially growing TG1 culture $\left(\mathrm{OD}_{600}\right.$ of 0.6 to 0.8$)$. In addition, $4 \mathrm{ml}$ of this culture were added to the immuno-tube to collect any remaining phages. Both cultures were incubated for 30 minutes at $37^{\circ} \mathrm{C}$ in a water bath without shaking to allow infection. The cultures were then pooled and serial dilutions were plated on TYE containing $100 \mu \mathrm{g} / \mathrm{ml}$ ampicillin and $1 \%$ glucose for titer determination. The remaining culture was centrifuged at $3,300 \mathrm{~g}$ for 10 minutes and resuspended in $1 \mathrm{ml}$ of $2 \mathrm{xTY}$ and plated on a large Nunc Bio-Assay dish of TYE containing $100 \mu \mathrm{g} / \mathrm{ml}$ ampicillin and $1 \%$ glucose. After overnight incubation at $30^{\circ} \mathrm{C}, 5-6 \mathrm{ml}$ of $2 \times \mathrm{TY}$ and $15 \%$ glycerol was added to the Bio-Assay dish and the cells were suspended with a glass spreader. The bacteria were then incubated in 2 XTY medium containing ampicillin $(100 \mu \mathrm{g} / \mathrm{ml})$ and $1 \%$ glucose at $37^{\circ} \mathrm{C}$ with shaking until the $\mathrm{OD}_{600}$ reached 0.5 .

Subsequent selection of primarily purified phages was also done using Nunc immunotubes (maxisorp®) coated overnight with $4 \mathrm{ml}$ of monomeric and pentameric $\operatorname{EtxB}(10 \mu \mathrm{g} / \mathrm{ml})$ at room temperature in $50 \mathrm{mM}$ carbonate buffer, $\mathrm{pH}$ 9.6 .

Preparation of monoclonal phages Monoclonal phages were prepared by inoculating individual colonies from the TYE plates into $100 \mu \mathrm{l}$ of $2 \mathrm{xTY}$ containing $100 \mu \mathrm{g} / \mathrm{ml}$ ampicillin and $1 \%$ glucose in 96 well plates and grown at $37^{\circ} \mathrm{C}$ overnight with shaking at $300 \mathrm{rpm} .2 \mu 1$ of 
each well from this plate was transferred to a second 96-well plate containing $200 \mu 1$ of 2 xTY with $100 \mu \mathrm{g} / \mathrm{ml}$ ampicillin and $1 \%$ glucose per well and incubated at $37^{\circ} \mathrm{C}$ for $1 \mathrm{~h}$. Then $25 \mu \mathrm{l} 2 \mathrm{xTY}$ containing $100 \mu \mathrm{g} / \mathrm{ml}$ ampicillin, $1 \%$ glucose and $10^{9}$ PFU VCS-M13 helper phage was added to each well. After $90 \mathrm{~min}$ incubation at $37^{\circ} \mathrm{C}$ the plate was spun at $(10 \mathrm{~min}$ at $1,800 \mathrm{~g})$, the pellet was resuspended in $200 \mu 1$ 2xTY containing $100 \mu \mathrm{g} / \mathrm{ml}$ ampicillin and $50 \mu \mathrm{g} / \mathrm{ml}$ kanamycin and incubated overnight at $30^{\circ} \mathrm{C}$ with shaking. The plate was spun $(10 \mathrm{~min}$ at $1,800 \mathrm{~g})$ and $100 \mu \mathrm{l}$ of the supernatant was used in phage-ELISA.

\section{Enzyme-linked immunosorbent $\underline{\text { assays }}-$}

The production and purification of recombinant pentameric B-subunit $\left(\mathrm{EtxB}_{5}\right)$ has been described previously (Amin $\underline{e t} \underline{a l} ., 1995$ ).. For the $\mathrm{G}_{\mathrm{M} 1^{-}} \mathrm{EtxB}$ ELISA, microtiter plates (Immulon 1, Dynatech) were coated with $\mathrm{G}_{\mathrm{M} 1}$-ganglioside $(0.2 \mu \mathrm{g} / \mathrm{ml})$ overnight and $0.2 \mu \mathrm{g} / \mathrm{ml} \mathrm{EtxB}{ }_{5}$ in PBS was added $1 \mathrm{hr}$ before the assay. Plates were blocked with $1 \%$ bovine serum albumin (BSA) and antibody binding to $\mathrm{G}_{\mathrm{M} 1}$ captured EtxB was detected using a goat anti-mouse-horse radish peroxidase conjugate (Jackson Immunoresearch Laboratories).

For direct binding experiments not involving the capture of $\operatorname{EtxB}_{5}$ by $\mathrm{G}_{\mathrm{M} 1}$. EtxB $\mathrm{E}_{5}$ and $\operatorname{EtxB}_{1}\left(\mathrm{EtxB}_{5}\right.$ boiled for $\left.5 \mathrm{~min}\right)$ were coated directly onto the plates in PBS for $1 \mathrm{hr}$ at room temperature and blocked with BSA buffer as above. To confirm that plates were coated with $\mathrm{EtxB}_{5}$ or $\mathrm{EtxB}_{1}$ we used the monoclonal antibodies against pentamer (LDS16) and monomer (LDS47) as controls

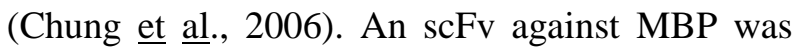
also used as an internal positive control for the detection system in ELISA (kindly provided by Dr. K. Gough, Department of Biology, University of Leicester (Gough $\underline{e t} \underline{a l} .$, 1999)) although data for this is not shown for reasons of clarity. For the phage-ELISA, $10 \mu 1$ (about $10^{10} \mathrm{PFU}$ ) of polyethylene glycol-precipitated polyclonal phage antibodies from each round of selection (made up to $100 \mu 1$ with blocking buffer) or $100 \mu 1$ of culture supernatant of super-infected TG1 cells containing monoclonal phage antibodies was applied to the coated and blocked plates and incubated for 
$90 \mathrm{~min}$ at room temperature. Plates were washed three times with PBS containing 0.05\% Tween20 and three times with PBS. Phages were detected by using the Detection Module Recombinant Phage Antibody System (Pharmacia, Uppsala, Sweden) according to the manufacturer's recommendations.

To test for secreted scFvs, E. coli supernatants were applied to the coated and blocked plates and incubated for $1.5 \mathrm{~h}$. Then the anti-c-myc monoclonal antibody (9E10, 1/50 dilution of hybridoma supernatant) was added and incubated for $1.5 \mathrm{~h}$ followed by $1 \mathrm{~h}$ incubation with goat anti-mouse IgG conjugated to horseradish peroxidase (Jackson Immunoresearch Laboratories). The substrate reaction was carried out for $1 \mathrm{~h}$ in the dark. The reaction was stopped by addition of $1 \mathrm{M} \mathrm{H}_{2} \mathrm{SO}_{4}$ and the absorbance was measured at $450 \mathrm{~nm}$.

Inhibition of scFv-B5.2.14 binding was analyzed by first incubating $\operatorname{EtxB}_{1} / \operatorname{EtxB}_{5}$ with either B5.2.14 (E. coli supernatant containing the secreted $\mathrm{scFv}$ ) or LDS16 (diluted 1/50 in PBS) for
$1 \mathrm{~h}$ at room temperature before adding to the ELISA plates directly coated with $\mathrm{EtxB}_{5}$ or $\mathrm{G}_{\mathrm{M} 1^{-}}$ captured $\mathrm{EtxB}_{5}$ and subsequently blocked with $1 \%$ milk powder in PBS. The inhibition was calculated using:

$$
I=1-\frac{O D(\text { competitor })}{O D(\text { no competitor })}
$$

For quantification of the amount of $\mathrm{EtxB}_{5}$ bound to the ELISA plates for each test sample, optical density readings corresponding to dilutions located on the linear part of the curve were compared with the dilution of an $\mathrm{EtxB}_{5}$ standard $(1 \mu \mathrm{g} / \mathrm{ml}$, diluted 2-fold), giving the same optical density reading.

Correction of the scFv-B1.3.9 gene - The nonsense mutation identified in the $\mathrm{V}_{\mathrm{H}}$ region of scFv-B1.3.9 was corrected by SOE-PCR using the primers pSOEp1 (5' AAA TTC CAA GAC AGA GTC ACC ATT ACC AG 3'), pSOEp2 (5' GGT GAC TCT GTC TTG GAA TTT CTG TGC GTA GTT 3'), pGSRp3 (5' TCC GCC TGA ACC GCC TCC AC 3') and LMB3 (5' CAG GAA ACA GCT ATG AC 3'). The SOE-PCR product was gel- 
purified and digested with SfiI and XhoI. The corresponding region of pHEN2-scFv-B1.3.9 as excised and the gel-purified vector-fragment (V-F) was ligated to the digested SOE-PCR product. The ligated product was transformed into electrocompetent XL1-blue cells. Ampicillin-resistant colonies were isolated for further analysis.

Structural analysis - The variable regions of B1.3.9 and B5.2.14 were sequenced and their deduced amino-acid sequences were analyzed by aligning them to the human germline sequences (http://imgt.cines.fr/). The complementary determining regions were assigned according to Chothia (Chothia $\underline{e t} \underline{a l} ., 1992)$. 3D models of the scFvs were generated by homology-based modeling using the SWISS-model and WAM (Chothia $\underline{\text { et }} \underline{\text { al., }}$ 1992). Graphic representations of the 3D molecular features were generated with the Swiss-PdbViewer v3.7 software package (Guex $\underline{e t}$ $\underline{a l .} ., 1997)$ using previously published structures of $\mathrm{CtxB}_{5}$ (Merritt $\underline{\text { et }} \underline{\text { al. }}$. 1997) and $\mathrm{EtxB}_{5}$ (Sixma $\underline{e t}$ al., 1992). Electrostatic molecular surface potentials were generated using the eF-site (http://ef-site.hgc.jp/)

$$
\underline{\text { Expression }} \text { of soluble } \underline{s c F v}-\underline{\text { E. coli }}
$$

HB2151 was infected with individual phages for $30 \mathrm{~min}$ at $37^{\circ} \mathrm{C}$ and then plated onto TYE plates containing $100 \mu \mathrm{g} / \mathrm{ml}$ ampicillin and incubated overnight at $37^{\circ} \mathrm{C}$. Single colonies were inoculated in 2 xTY medium $(16 \mathrm{~g} / \mathrm{L}$ tryptone, $10 \mathrm{~g} / \mathrm{L}$ yeast extract and $5 \mathrm{~g} / \mathrm{L} \mathrm{NaCl}$ ) containing $100 \mu \mathrm{g} / \mathrm{ml}$ ampicillin and $1 \%$ glucose. $\mathrm{scFv}$ expression was induced with $1 \mathrm{mM}$ isopropyl $\beta$-D-thiogalactoside (IPTG) when the cultures reached an $\mathrm{OD}_{600}=0.9$. The cultures were further incubated overnight at $30^{\circ} \mathrm{C}$ and the culture supernatants containing the secreted scFvs were collected by centrifugation at $3,000 \mathrm{rpm}$ for $15 \mathrm{~min}$ for subsequent analysis.

Periplasmic extracts were prepared by resuspending the bacterial pellet in $10 \mathrm{ml}$ of icecold PBS, pH 7.2 containing polymyxi B sulphate $(1,000 \mathrm{units} / \mathrm{ml})$ and incubated on ice for $15 \mathrm{~min}$. Samples were centrifuged for $10 \mathrm{~min}$ at $4^{\circ} \mathrm{C}$ at 3,000 rpm and $100 \mu \mathrm{l}$ of the supernatants were used for ELISA. 
$\underline{\text { SDS-polyacrylamide Gel Electrophoresis and }}$

\section{Western blot-}

EtxB5 $(8 \mu \mathrm{g})$, boiled (5min) EtxB5 (EtxB1 $-8 \square \mathrm{g})$ and boiled/reduced (in 30mM dithiothreitol) were loaded into SDS-polyacrylamide $14 \%$ (w/v) gels for electrophoresis on a Bio-Rad Protean II system. After SDS-PAGE, gels were transferred to nitrocellulose paper with transfer buffer in a BioRad Trans-Blot apparatus for $45 \mathrm{~min}$ to permit efficient transfer. Non-specific binding sites were blocked overnight in PBS containing 5\% (w/v) Marvel. Blots were washed in PBS containing $0.05 \% \mathrm{v} / \mathrm{v}$ Tween 20 (PBS-T) for $3 \mathrm{x}$ 10min with agitation. B5.2.14 supernatant (approximately $2 \square \mathrm{g} / \mathrm{ml}$ ) was used neat for $1 \mathrm{~h}$ at room temperature with agitation. Blots was washed as previously and incubated with goat anti-mouse $\mathrm{IgG}$ conjugated to horseradish peroxidase diluted 1:2000 for $1 \mathrm{~h}$. The blots were then washed and developed with an enhanced chemiluminescence system (ECL, Amersham) for 10min.

\section{RESULTS}

Selection of phage-displayed $\underline{s c F v s}-$ The Griffin.1 human scFv library was expanded and the phagemids selected by solid phase panning using antigen directly coated to test tubes (immuno-tubes). After initial panning, antigenbinding phages were collected and expanded following re-infection of TG1 bacterial cells. This cycle of selection and expansion was repeated 3 times following selection for phagemids binding to either $\operatorname{EtxB}_{1}$ or $\mathrm{EtxB}_{5}$. Resulting enrichment was assessed using ELISA against plates coated with

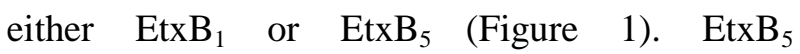
specificities were in greater abundance in the library and by the third round the majority of colonies tested had reactivity against $\mathrm{EtxB}_{5}$. It was clear that the library had less reactivity against EtxB $B_{1}$ and even after 3 rounds of selection against $\operatorname{EtxB}_{1}$ there was markedly less reactivity seen in the isolated phages (Figure 1). 
Analysis of selected monoclonal phages

- The clones displaying the highest reactivities

were then transfected into $E$. coli TG1 cells for scale up to confirm the antigen-binding properties and follwing the expansion of the $3 \mathrm{EtxB}_{1}$ selected clones in TG1 cells only phage-displayed B1.3.9 showed consistent reactivity in ELISA (Figure 2A). In terms of specificity B1.3.9 also showed reactivity against $\mathrm{EtxB}_{5}$ coated directly to plastic (Figure 2A). However, following transfection of the phagemid into permissive HB2151 bacterial cells, no soluble $\mathrm{scFv}$ was present (Figure 2B) (summarized in Table 1). This is discussed later in relation to the sequence analysis.

Two of the 4 phagemid colonies expanded in TG1 cells from the selected EtxB ${ }_{5}$ panning showed consistent results in ELISA (Figure 2A) and both B5.2.11 and B5.2.14 both gave a higher ELISA signal for $\mathrm{EtxB}_{5}$ over EtxB ${ }_{1}$. However, only the $\mathrm{EtxB}_{5}$ specific $\mathrm{scFv}$ (B5.2.14) was secreted following transfection into HB2151 cells (Figure 2B).

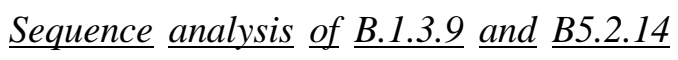
$\underline{s c F v}$ - The inserts of scFvs that were specific for $\mathrm{EtxB}_{1}$ or $\mathrm{EtxB}_{5}(\mathrm{~B} 1.3 .9$ and B5.2.14) were sequenced to assess their germline alignment specificities compared to the published V-BASE directory. The phage display library was generated from human $\mathrm{VH}, \mathrm{V} \lambda$ and $\mathrm{V} \kappa$ germline exons and its generation and composition has been described and analyzed in great detail (Tomlinson $\underline{e t} \underline{a l}$, 1992; Nissim $\underline{\text { et }} \underline{\text { al. }}$, 1994). A detailed comparison with the identified $\mathrm{scFv}$-clones was therefore done to identify unusual sequence features or artifacts that may have been acquired during the course of the experiments. We therefore determined the DNA sequences of scFv-B1.3.9 and scFv-B5.2.14 (Figure 3) and identified the corresponding germline exons.

The VL of ScFv-B5.2.14 belongs to the V $\lambda$-I subgroup and has highest homology with germline segment DP-L3. There is one single nucleotide change leading to a conservative $\mathrm{Gly}^{95 \mathrm{~b}} \rightarrow \mathrm{Ala}^{95 \mathrm{~b}}$ mutation located in the CDR-L3. The VH of scFv-B5.2.14 belongs to the VH-I 
subgroup and shows closest homology to the germline segment DP-14. There is also one conservative mutation leading to a $\operatorname{Tr}^{103} \rightarrow \mathrm{Leu}^{103}$ change. $\operatorname{Trp}^{103}$ is a very strongly conserved residue and is found in all 6 germline segments of the joining element. Both observed mutations are exactly at the junctions where the $0-3$ and $4-12$ amino acid long random insertions have been made (Nissim et $\underline{\text { al., }}$ 1994). This suggests that these mutations have likely resulted from the initial generation of the recombinant antibody genes and were not acquired during panning and selection.

The VL of scFv-B1.3.9 belongs to the V $\lambda$ III subgroup showing highest homology to the germline segment DP-L16 (Figure 3). There are three amino acid changes, all of which are not critical in terms of structural aspects. The $\mathrm{Thr}^{5} \rightarrow \mathrm{Ile}^{5}$ change is located in framework- 1 and is a surface exposed residue. The $\mathrm{Asn}^{95 \mathrm{a}} \rightarrow \mathrm{Thr}^{95 \mathrm{a}}$ and $\mathrm{His}^{95 \mathrm{~b}} \rightarrow \mathrm{Val}^{95 \mathrm{~b}}$ changes are located in CDR-L3 and are likely part of the paratope of $\mathrm{scFv}-\mathrm{B} 1.3 .9$. These residues are also located at the junction where the 0-3 amino acid long random insertions have been made. The VH of scFv-B1.3.9 belongs to the VH-I subgroup having highest homology to germline segment DP-4. Three mutations have been identified. The changes from $\mathrm{Met}^{2} \rightarrow \mathrm{Val}^{2}$ and $\mathrm{Met}^{89} \rightarrow \mathrm{Val}^{89}$ are conservative. Valine is preferentially found in the other germline segments of the VH-I subgroup and can therefore be judged to be a non-problematic change. The third mutation, however, is a critical nonsense mutation leading to an amber stop codon at position 64 $\left(\mathrm{Gln}^{64} \rightarrow \mathrm{amber}^{64}\right)$. This explains why the expression of soluble scFv was not successful while strong reactivity was observed for the phagedisplayed scFv-B1.3.9 (Figure 2B). The phagedisplayed $\mathrm{scFv}$ is expressed in E. coli TG1, which is a suppressor strain to allow the expression of a fusion-protein between the pIII and the scFv. TG1 can introduce glutamine at amber stop codons, thus restoring the authentic germline residue at position H64. In contrast, E. coli strain HB2151 used for production of soluble $\mathrm{scFv}$ is a non-suppressor 
strain. As a result, no functional soluble form of scFv-B1.3.9 is produced in this strain.

In order to overcome the problem of the nonsense mutation introduced at position $\mathrm{H} 64$ of scFv-B1.3.9, preventing the expression of a soluble form of the $\mathrm{scFv}$, it was converted to the designated germline sequence using SOE-PCR (see Methods). The corrected clone was sequenced to confirm the introduction of the $\mathrm{Gln}^{64}$ coding sequence (data not shown) and this new clone was designated scFv-B1.3.9 ${ }^{c}$.

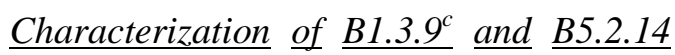
- Nine ampicillin resistant colonies were selected for production of soluble scFv-B1.3.9 ${ }^{\mathrm{c}}$ (Figure 4). All clones expressed functional scFvs that bound to $\mathrm{EtxB}_{1}$ showing that the acquired nonsense mutation was successfully and properly corrected. We then went on to analyze the binding specificity of $\mathrm{scFv}-\mathrm{B} 1.3 .9^{\mathrm{c}}$ by comparing the binding against EtxB $_{1}$ and directly coated EtxB $\mathrm{B}_{5}$ As was previously observed for the phage-displayed B1.3.9 (Figure 2A), the soluble $\mathrm{scFv}-\mathrm{B} 1.3 .9^{\mathrm{c}}$ exhibited equivalent binding activity against both
$\mathrm{EtxB}_{1}$ and directly coated $\mathrm{EtxB}_{5}$ suggesting it recognizes a shared epitope. However scFvB1.3.9 $9^{\mathrm{C}}$ was shown not to bind to $\mathrm{EtxB}_{5}$ captured to $\mathrm{G}_{\mathrm{M} 1}$-ganglioside (Figure 4) suggesting the epitope becomes inaccessible when the complex is formed. We then used a recently described, wellcharacterized conformation specific monoclonal antibody for comparison to B5.2.14 (Chung $\underline{\text { et }} \underline{\text { al., }}$, 2006). LDS16 is a neotope-specific monoclonal antibody that binds to directly coated as well as to $\mathrm{G}_{\mathrm{M} 1}$-ganglioside captured $\mathrm{EtxB}_{5}$ and does not recognize $\mathrm{EtxB}_{1}$. As in our previous study we looked at the specificity of the antibodies by concentration dependent inhibition assays (Figure 5). As we described previously LDS16 binding to coated $\mathrm{EtxB}_{5}$ was inhibited in the presence of soluble $\mathrm{EtxB}_{5}$. However, while B5.2.14 binding was partially inhibited, this only reached a maximum level of $10 \%$ (Figure 5A). As expected, LDS16 binding to $\mathrm{G}_{\mathrm{M} 1}$ captured $\mathrm{EtxB}_{5}$ was not inhibited by $\operatorname{EtxB}_{1}$ and there was no binding of B5.2.14 to $\mathrm{G}_{\mathrm{M} 1}$ captured $\mathrm{EtxB}_{5}$ (Figure 5B). Lastly, there was no inhibition of either LDS16 or B5.2.14 
binding to $\mathrm{EtxB}_{5}$ coated plates by $\mathrm{EtxB}_{1}$ produced by boiling $\mathrm{EtxB}_{5}$ (Figure $5 \mathrm{C}$ ), while there was a marked contrast in the results obtained by using boiled and reduced $\mathrm{EtxB}_{5}$ (Figure 5D). Here, EtxB (DTT) markedly inhibited the binding of B5.2.14 to $\mathrm{EtxB}_{5}$ coated plates while, as shown previously, LDS16 binding was unaffected. This was confirmed using western blotting, which showed that B5.2.14 only reacted with DTT reduced $\mathrm{EtxB}_{5}$, and not with boiled $\mathrm{EtxB}_{5}$ (Figure 5E). These results serve to illustrate the rather subtle but distinctive differences of the binding specificities between these two recombinant antibodies.

\section{DISCUSSION}

Here we have reported the successful isolation of scFvs against EtxB by phage display. As in previous studies with conventional mAbs (Chung et al., 2006) we have set out to define individual scFvs that can differentiate between different forms of EtxB.
We and others have previously used a combination of ELISA and SDS-PAGE to study the kinetics of both EtxB and CtxB assembly (Ruddock $\underline{e t} \underline{a l} ., 1995)$. In later experiments we used a combination of these techniques and tryptophane fluorescent spectroscopy to elucidate a kinetic model of how toxin assembly in vitro may take place (Lesieur $\underline{e t} \underline{a l .} ., 2002)$. It was shown that assembly of $\mathrm{CtxB}_{5}$ occurs via ordered formation of oligomeric species and that they can equally go through two alternative pathways to form the fully assembled pentamer. In addition, other experiments investigating $\mathrm{A} / \mathrm{B}$ subunit assembly have suggested that holotoxin formation requires the A-subunit to interact with a B-subunit assembly intermediate rather than with fully assembled $\mathrm{CtxB}_{5}$ (Hirst, 1995).

Our studies using conventional mAbs have identified epitopes potentially important for investigating pentameric subunit assembly but these have limitations for use in studying assembly in vivo (Chung et al., 2006). Recombinant singlechain Fv antibodies potentially offer a great 
advantage in this respect. Other studies using monoclonal scFvs against $(\underline{H}$. pylori $)$ urease (Reiche et al., 2002) and hepatitis B virus surface antigen (HBsAg) (Zhang $\underline{e t} \underline{\text { al., 2006) have }}$ highlighted the advantage of this approach. In the first instance anti-urease antibodies derived from H. pylori infected patients have helped to define important antigenic epitopes involved with disease recovery (Reiche et al., 2002) and in the second instance anti-HBsAg scFvs are being used to develop strategies to block viral entry (Zhang $\underline{e t}$ al., 2006). In addition these anti-HBsAg scFvs fused with interferon can be used as a reagent in the therapy of chronic hepatitis B (Zhang $\underline{e t} \underline{a l}$., 2006). These newly prepared anti-EtxB scFvs open up considerable scope for extending earlier studies by potentially enabling the interaction of antibodies with toxin assembly intermediates to be carried out both in vitro and in vivo.

Selection was performed against two different forms of EtxB, the heat dissociated disassembled monomeric form $\mathrm{EtxB}_{1}$ and the assembled pentameric form $\mathrm{EtxB}_{5}$. After two to three rounds of panning monoclonal phage-ELISA showed that phagemid clones were selected that exhibited binding specificity to $\mathrm{EtxB}_{1}$ or $\mathrm{EtxB}_{5}$ (Figure 2). Further characterization of individual clones allowed us to focus on one phagemid with specificity for $\operatorname{EtxB}_{1}(\mathrm{scFv}-\mathrm{B} 1.3 .9)$ and one with specificity for $\mathrm{EtxB}_{5}$ (scFv-B5.2.14) (Table 1, Figure 2). We should note that although no isolated scFvs showed specificity for $\mathrm{EtxB}_{1}$ without cross-reactivity to directly coated $\mathrm{EtxB}_{5}$ we do have some preliminary evidence (data not shown) that such scFvs may be present in the library.

Since both B5.2.14 and B1.3.9 ${ }^{\mathrm{c}}$ do not bind to $\mathrm{G}_{\mathrm{M} 1}$-ganglioside captured $\mathrm{EtxB}_{5}$ it could be hypothesized that they recognize an epitope that overlaps with the $\mathrm{G}_{\mathrm{M} 1}$-ganglioside binding site or an epitope that is located at the bottom surface of the pentameric EtxB. The surface potential of both $\mathrm{EtxB}_{5}$ and $\mathrm{CtxB}_{5}$ in this region is highly basic to facilitate binding to the negatively charged cell surface (Figure 6B). Thus, it is rational to expect that an antibody binding in this region would have 
an antigen-binding site with a negative surface potential. However, inspection of the amino-acid composition of the CDRs (Figure 3) shows a relatively high preponderance of basic residues for both B1.3.9 ${ }^{\mathrm{c}}$ and B5.2.14. Theoretical calculations of the Fv iso-electric points yielded 9.8 for $\mathrm{B} 1.3 .9^{\mathrm{c}}$ and 9.6 for B5.2.14. To further support this notion we generated homology models for both scFvs to specifically look for the charge distribution in the paratope.

Both paratopes exhibit highly basic surfaces (Figures 6C and D) that make it unlikely that either B1.3.9 ${ }^{\mathrm{C}}$ or B5.2.14 would recognize an epitope located at the bottom side of pentameric EtxB although this cannot be excluded. Interestingly, the peptides identified by Marcel $\underline{e t}$ al. (in this issue) were also highly basic, suggesting that similar binding regions were targeted by both approaches. Moreover, binding of the phage-displayed peptides to $\mathrm{EtxB}_{5}$ was not inhibited by soluble $\mathrm{G}_{\mathrm{M} 1}$-ganglioside and soluble $\mathrm{EtxB}_{5}$ bound to phages dotted onto nitrocellulose. Thus steric hindrance offers no complete or satisfying explanation for the observed lack of binding towards $\mathrm{G}_{\mathrm{M} 1}$-ganglioside captured $\mathrm{EtxB}_{5}$. The specificity of $\mathrm{B} 1.3 .9^{\mathrm{c}}$ is, in fact, strongly reminiscent of the conventional mAb LDS47 against an $\mathrm{N}$-terminal region of $\mathrm{EtxB}_{1}$ (Figure $6 \mathrm{~A}$ ) that we previously described which also bound to directly coated $\mathrm{EtxB}_{5}$ (Amin $\underline{\text { et }} \underline{\text { al. }}$. 1995).

The fine specificity of B5.2.14 was investigated using competition ELISA assays with the conventional mAb LDS16 as a comparison (Figure 5). This gave an intriguing result. Firstly, soluble $\mathrm{EtxB}_{5}$ inhibition of binding to directly coated $\mathrm{EtxB}_{5}$ was only marginal (Figure $5 \mathrm{~A}$ ). We can think of only two possible explanations for this $10 \%$ maximal inhibition: a) the existence of different conformational states of the epitope and b) the presence of disassembled intermediates. Since disassembled intermediates would only constitute a small fraction of the total protein because of the high intrinsic stability of the pentameric form we believe the existence of different conformational states of the epitope to be more likely. We have previously hypothesized that 
a conformational change occurs in the protein upon binding to a solid surface exposing an epitope not normally accessible in the fully folded conformation (Lesieur $\underline{e t} \underline{a l}$. , 2002). Similar conclusions have been drawn from studies with the $\beta 2$ subunit of $\underline{E}$. coli tryptophan synthase (Friguet et $\underline{\text { al., }}$ 1984). Secondly, although, as we showed earlier (Figure 2B), there was no binding in ELISA of scFv-B5.2.14 to denatured (boiled) $\mathrm{EtxB}_{1}$, in Figure 5D we clearly show that DTT reduced/denatured $\mathrm{EtxB}_{1}$ strongly inhibits binding to $\mathrm{EtxB}_{5}$. These results were confirmed by western blotting, which not only gave an appropriate band $(\mathrm{Mr}=12,000)$ for the denatured/boiled EtxB but also gave a faint band $(\mathrm{Mr}=43,000)$ consistent with $\mathrm{EtxB}_{5}$ (Figure 5E). Thus, this is almost certainly a conformation dependent epitope (cryptotope) probably of a folding intermediate structure (Figure 6A) and could be an ideal target for studying pentamer formation $\underline{i n} \underline{s i t u}$. It would be interesting to repeat these inhibition experiments with $\mathrm{B} 1.3 .9^{\mathrm{c}}$ to look at its finer specificity.

In conclusion, this paper reports the generation of two scFvs with differential binding specificities that may provide useful molecular probes for studying EtxB pentamer assembly. The availability of such reagents opens up key areas for the development of new therapeutics that can block toxin formation in situ and thus be used for the treatment of diarrhoeal diseases caused by E. coli and V. cholera. 


\section{ACKNOWLEDGEMENTS}

We would like to thank Dr. Andrew Griffiths for providing the Griffin.1 human scFv library (MRC, Cambridge, UK). Work on delineating models of CtxB assembly (Lesieur $\underline{\underline{t}} \underline{\underline{a l}}$.) and initial studies on screening the Griffin.1 library was kindly funded by the BBSRC (7/C10265)

\section{REFERENCES}

Aman, A. T., Fraser, S., Merritt, E. A., Rodigherio, C., Kenny, M., Ahn, M., Hol, W. G., Williams, N. A.,Lencer, W. I., and Hirst, T. R. (2001) Proc Natl Acad Sci U S A 98(15), 8536-8541

Amin, T., and Hirst, T. R. (1994) Protein Expr Purif 5(2), 198-204

Amin, T., Larkins, A., James, R. F., and Hirst, T. R. (1995) J Biol Chem 270(34), 20143-20150

Bost, K. L., and McGhee, J. R. (1996) J Infect Dis 173(3), 627-635

Chothia, C., Lesk, A. M., Gherardi, E., Tomlinson, I. M., Walter, G., Marks, J. D., Llewelyn, M. B., and Winter, G. (1992) J Mol Biol 227(3), 799-817

Chung, W. Y., Carter, R., Hardy, T., Sack, M., Hirst, T. R., and James, R. F. (2006) J Biol Chem 281(51), 39465-39470

Echeverria, P., Seriwatana, J., Taylor, D. N., Yanggratoke, S., and Tirapat, C. (1985) Am J Trop Med Hyg 34(3), 547-554

Fishman, P. H., Moss, J., and Osborne, J. C., Jr. (1978) Biochemistry 17(4), 711-716

Fishman, P. H., Pacuszka, T., Hom, B., and Moss, J. (1980) J Biol Chem 255(16), 7657-7664

Fraser, S. A., de Haan, L., Hearn, A. R., Bone, H. K., Salmond, R. J., Rivett, A. J., Williams, N. A., and Hirst, T. R. (2003) Infect Immun 71(3), 1527-1537

Friguet, B., Djavadi-Ohaniance, L., and Goldberg, M. E. (1984) Mol Immunol 21(7), 673-677 
Gough, K. C., Li, Y., Vaughan, T. J., Williams, A. J., Cockburn, W., and Whitelam, G. C. (1999) J Immunol Methods 228(1-2), 97-108

Griffiths, A. D., Williams, S. C., Hartley, O., Tomlinson, I. M., Waterhouse, P., Crosby, W. L., Kontermann, R. E., Jones, P. T., Low, N. M., Allison, T. J., and et al. (1994) Embo J 13(14), 3245-3260

Guex, N., and Peitsch, M. C. (1997) Electrophoresis 18(15), 2714-2723

Hirst, T. R. (1995) Bacterial Toxins and Virulence Factors in Disease. In: Moss, J., Vaughan, M., Iglewski, B., and Tu, A.T. (ed). Handbook of Natural Toxins, Marcel Dekker Inc., New York

Holmgren, J., Lonnroth, I., Mansson, J., and Svennerholm, L. (1975) Proc Natl Acad Sci U S A 72(7), 2520-2524

Kuziemko, G. M., Stroh, M., and Stevens, R. C. (1996) Biochemistry 35(20), 6375-6384

Lesieur, C., Cliff, M. J., Carter, R., James, R. F., Clarke, A. R., and Hirst, T. R. (2002) J Biol Chem 277(19), 16697-16704

Luross, J. A., Heaton, T., Hirst, T. R., Day, M. J., and Williams, N. A. (2002) Arthritis Rheum 46(6), $1671-1682$

Merritt, E. A., Sarfaty, S., Jobling, M. G., Chang, T., Holmes, R. K., Hirst, T. R., and Hol, W. G. (1997) Protein Sci 6(7), 1516-1528

Nissim, A., Hoogenboom, H. R., Tomlinson, I. M., Flynn, G., Midgley, C., Lane, D., and Winter, G. (1994) Embo J 13(3), 692-698

Reiche, N., Jung, A., Brabletz, T., Vater, T., Kirchner, T., and Faller, G. (2002) Infect Immun 70(8), 41584164

Ruddock, L. W., Ruston, S. P., Kelly, S. M., Price, N. C., Freedman, R. B., and Hirst, T. R. (1995) J Biol Chem 270(50), 29953-29958

Shevach, E. M. (2000) Annu Rev Immunol 18, 423-449 
Sixma, T. K., Pronk, S. E., Kalk, K. H., van Zanten, B. A., Berghuis, A. M., and Hol, W. G. (1992) Nature $355(6360), 561-564$

Sobel, D. O., Yankelevich, B., Goyal, D., Nelson, D., and Mazumder, A. (1998) Diabetes 47(2), 186-191

Takahashi, I., Marinaro, M., Kiyono, H., Jackson, R. J., Nakagawa, I., Fujihashi, K., Hamada, S.

Clements, J. D.,

Williams, N. A., Stasiuk, L. M., Nashar, T. O., Richards, C. M., Lang, A. K., Day, M. J., and Hirst, T. R. (1997)

Proc Natl Acad Sci U S A 94(10), 5290-5295

Tomlinson, I. M., Walter, G., Marks, J. D., Llewelyn, M. B., and Winter, G. (1992) J Mol Biol 227(3), 776-798

Truitt, R. L., Hanke, C., Radke, J., Mueller, R., and Barbieri, J. T. (1998) Infect Immun 66(4), 1299-1308

Turnbull, W. B., Precious, B. L., and Homans, S. W. (2004) J Am Chem Soc 126(4), 1047-1054

Weiner, H. L. (1997) Immunol Today 18(7), 335-343

Williams, N. A., Hirst, T. R., and Nashar, T. O. (1999) Immunol Today 20(2), 95-101

Wilson, A. D., Bailey, M., Williams, N. A., and Stokes, C. R. (1991) Eur J Immunol 21(10), 2333-2339

Yamamoto, T., Gojobori, T., and Yokota, T. (1987) J Bacteriol 169(3), 1352-1357

Zhang, J. L., Gou, J. J., Zhang, Z. Y., Jing, Y. X., Zhang, L., Guo, R., Yan, P., Cheng, N. L., Niu, B., and Xie, J. (2006) Hepatobiliary Pancreat Dis Int 5(2), 237-241 


\section{FIGURE LEGENDS}

Figure 1. Enrichment of polyclonal phages panned against EtxB monomers and pentamers. The polyclonal phages after the $1^{\text {st }}, 2^{\text {nd }}$ and $3^{\text {rd }}$ round of panning were tested for reactivity against monomeric and pentameric EtxB by direct phage-ELISA. Enrichment of scFvs against the pentamer was more efficient and the polyclonal phages exhibited only low reactivity against monomeric (boiled) EtxB. Phages selected for binding to monomeric EtxB, on the other hand, were enriched less efficiently and showed significant cross-reactivity against pentameric EtxB.

Figure 2. Binding specificities of monoclonal phages. (A) Several phages were selected for a large-scale phage preparation and their binding reactivities against different EtxB forms were analysed by ELISA. (B) Soluble scFvs were produced for 4 monoclonal phages and were analysed by ELISA.

Figure 3. Sequence analysis of scFv-B1.3.9 and scFv-B5.2.14. Sequences were aligned to the human germline segments reported in the V-BASE directory (http://vbase.mrc-cpe.cam.ac.uk/index.php). CDRs were assigned according to Cothia and are shown in bold. DP-L3, DP-L16, DP-14 and DP-4 are the designations of the germline sequences that most closely resemble those of the two scFvs and deviations are indicated. The insertion sites where the random sequences have been introduced are indicated by a gap.

Figure 4. ELISA for soluble $\mathrm{scFv}-\mathrm{B} 1.3 .9^{\mathrm{c}}$. Nine ampicillin-resistant colonies were picked and used for small scale expression of soluble $\mathrm{scFv}-\mathrm{B} 1.3 .9^{\mathrm{c}}$. Culture supernatants were analysed against monomeric (boiled) EtxB, directly coated $\mathrm{EtxB}_{5}$ and $\mathrm{G}_{\mathrm{M} 1}$-captured EtxB $\mathrm{B}_{5}$ by ELISA. 
Figure 5. Inhibition of the binding of B5.2.14 and LDS16 to different forms of soluble EtxB. (A) Plates were coated directly with $\mathrm{EtxB}_{5}(\mathrm{~B} 5 \mathrm{c})$ and probed with B5.2.14 or LDS16 and binding was inhibited by increasing concentrations of free $\mathrm{EtxB}_{5}$. (B) Plates were coated directly with $\mathrm{EtxB}_{5}(\mathrm{~B} 5 \mathrm{c})$ and boiled EtxB was used for competition. (C) Plates were coated directly with $\mathrm{EtxB}_{5}(\mathrm{~B} 5 \mathrm{c})$ and boiled and DTT treated EtxB was used as inhibitor. (D) Recognition of different forms of EtxB by scFv-B5.2.14 in Western-blot. Lane 1, Boiled EtxB ${ }_{1}$; Lane 2, EtxB ${ }_{5}$; Lane 3, Boiled and reduced (DTT) EtxB ${ }_{1}$. The single headed arrow denotes the positions of the monomeric form and the double headed arrow of the pentameric form.

Figure 6. 3D-visualization of molecular features of the pentameric B-subunit and scFv-B1.3.9 and scFv-B5.2.14. (A) Graphical illustration of antibody epitopes mapped onto proposed dimeric EtxB folding intermediates. The second EtxB protomer (cyan) is shown in two alternate conformations. In the folded form Pro $^{93}$ (single headed arrow) is in the cis state and $\mathrm{Cys}^{9}$ and $\mathrm{Cys}^{86}$ form a disulfide-bridge. The $\beta$ strand number 6 is shown in grey. In proposed partially folded dimers the cysteines are reduced and Pro ${ }^{93}$ is in the trans configuration (double headed arrow). Assembly can only proceed after isomerisation of Pro $^{93}$ and disulfide-bridge formation. MAb LDS102 recognizes to the C-terminal end of EtxB $_{1}$ and partially inhibits re-assembly (Chung et al 2006). The epitope of mAb LDS47 has been mapped to the Nterminal residues involving $\mathrm{Cys}^{9}$ (red and orange), but binding is independent of the redox-state. In contrast, scFv-B5.2.14 recognizes a different form and only binds to heat-denatured and reduced EtxB. ScFv-B1.3.9 recognizes a cryptotope that is not accessible in the assembled pentamer but becomes exposed after heat-denaturation. Similarly to LDS47 but contrary to scFv-B5.2.14, reduction of the disulfide-bridge is not a required for binding. (B) Visualisation of the surface potentials of EtxB $\mathrm{B}_{5}$ The bottom side is highly basic to facilitate binding to the negatively charged cell surface receptor. The top 
surface is characterised by a cluster of acidic residues comprising $\mathrm{Asp}^{22}, \mathrm{Glu}^{79}$ and the $\mathrm{C}$-terminus. Both scFv-B1.3.9 (C) and scFv-B5.2.14 (D) have high theoretical iso-electric points of 9.8 and 9.6 respectively. The surfaces potentials were mapped onto homology-modelled structures and are viewed from the top, looking down at the paratope. The antigen-binding site of scFvB1.3.9 is highly positively charged, suggesting binding to a negatively charged region. 
Table 1: Characterization of selected scFvs

\begin{tabular}{|c|c|c|c|c|}
\hline & Clone & OD 450nm & Scaled up phage prep & As soluble scFv \\
\hline \hline \multirow{3}{*}{$\begin{array}{c}\text { Monomer } \\
\text { (B1) }\end{array}$} & 3.1 & 0.4 & No reactivity & - \\
\cline { 2 - 5 } & 3.6 & 0.3 & No reactivity & - \\
\cline { 2 - 6 } & 3.9 & 1.0 & +ve for monomer (1.0) & No scFv found in C, P or S \\
\hline \multicolumn{5}{|c|}{} \\
\hline \multirow{3}{*}{$\begin{array}{c}\text { Pentamer } \\
\text { (B5) }\end{array}$} & 2.6 & 0.2 & Slight reactivity (0.1) & - \\
\cline { 2 - 6 } & 2.11 & 1.0 & Positive for Pentamer & scFv found in C only \\
\cline { 2 - 5 } & 2.14 & 1.0 & Positive for Pentamer & scFv found in C, P and S \\
\cline { 2 - 5 } & 2.15 & 0 & No reactivity & - \\
\hline
\end{tabular}

C - cytoplasmic fraction; P - periplasmic fraction; S - culture supernatant 


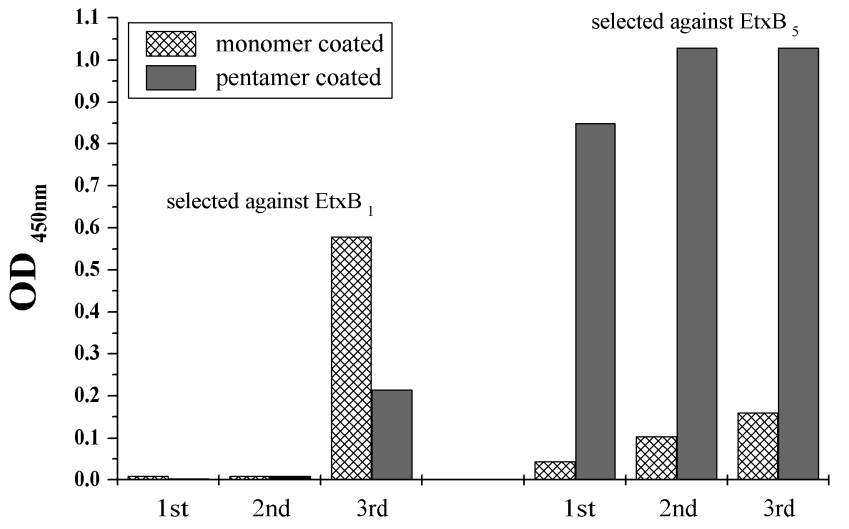




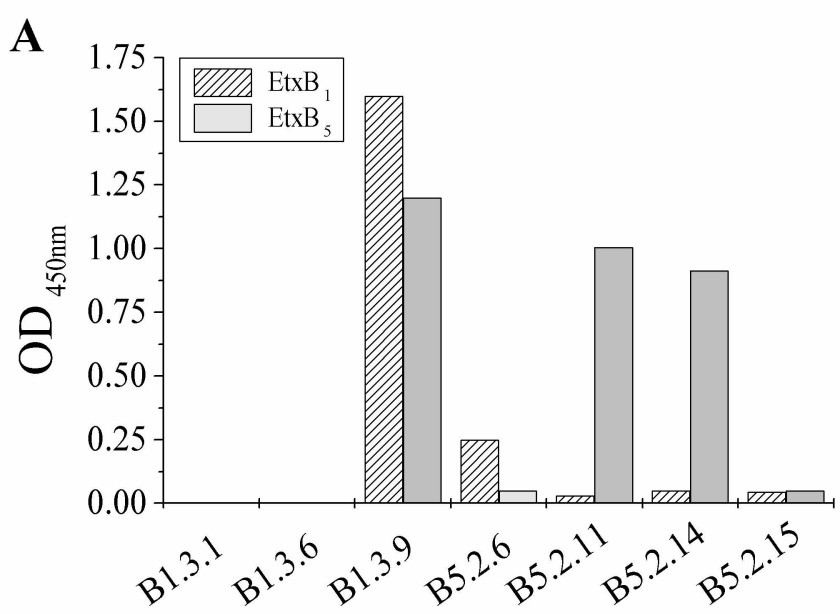

B

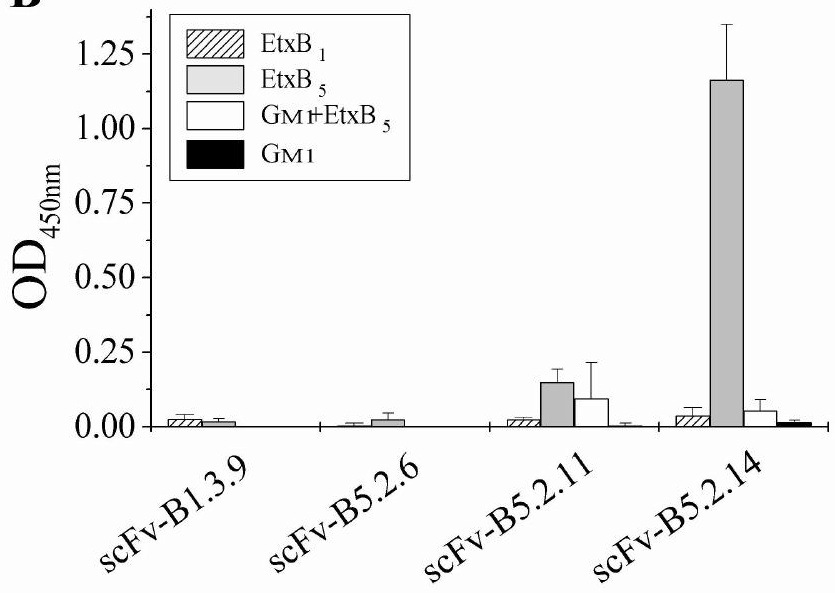




\section{light-chains}

$\mathrm{DP}-\mathrm{L} 3$

B5. 2.14

$\mathrm{B} 1.3 .9$

$\mathrm{DP}-\mathrm{L} 16$

$\mathrm{DP}-\mathrm{L} 3$

$\mathrm{B} 5.2 .14$

$\mathrm{B} 1.3 .9$

$\mathrm{DP}-\mathrm{L} 16$
CDR-L1

CDR-L2
QSVLTQPPSASGTPGQRVTISCSGSSSNIGSNYVYWYQQLPGTAPKLLI YRNNQRPS SSELIQDPAVSVALGQTVRITCQGDSL--RSYYASWYQQKPGQAPVLVI YGKNNRPS T
CDR-L3

G

GVPDRFSGSKSGTSASLAISGLRSEDEADYYCAAWDDSLSA LV FGGGTKLTVL GIPDRESGSSSGNTASLTITGAQAEDEADYYCNSRDSSGTV - - FGGGTKLTVL

heavy-chains

$\mathrm{DP}-14$

$\mathrm{B} 5.2 .14$

B1.3.9

$\mathrm{DP}-4$

$\mathrm{DP}-14$

$\mathrm{B} 5.2 .14$

B1.3.9

$\mathrm{DP}-4$

\section{$\mathrm{CDR}-\mathrm{H} 1$}

CDR-H2

QVQLVQSGAEVKKPGASVKVSCKASGYTFTSYGISWVRQAPGQGLEWMGWISAYNGNTNYAQ QVQLVQSGAEVKKTGSSVKVSCKASGYTFTYRYLHWVRQAPGQALEWMGWITPFNGNTNYAQ $\mathrm{M}$

$\mathrm{CDR}-\mathrm{H} 3$

KLQGRVTMTTDTSTSTAYMELRSLRSDDTAVYYCAR GRPPRPLRPSQV LGQGTLVTVSS KE*DRVTITRDRSMSTAYMELSSLRSEDTAVYYCAR GTLKRI----RN WGQGTLVTVSS $\bar{Q}$ 


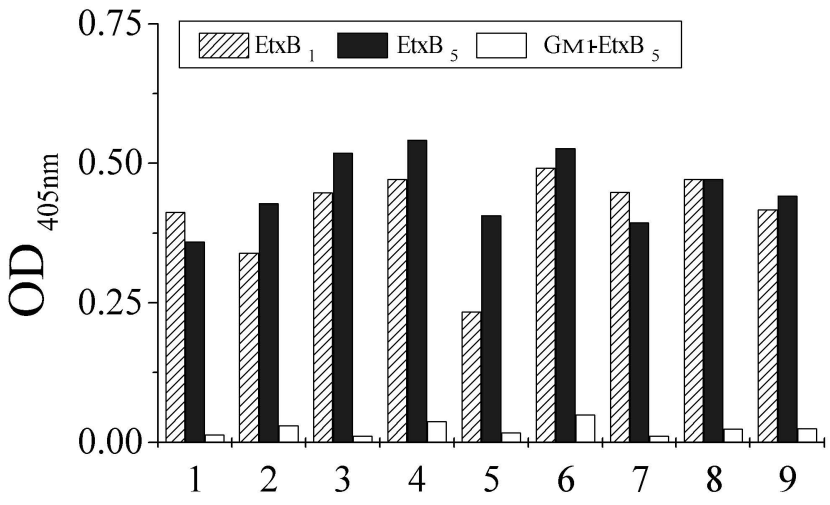




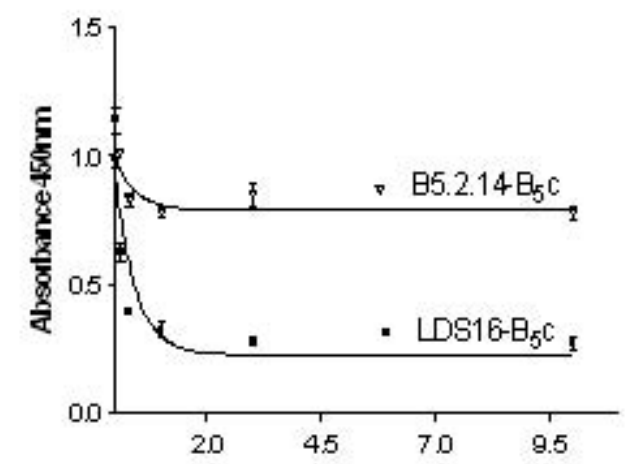

B5 concentration per wel - ugint

(C)

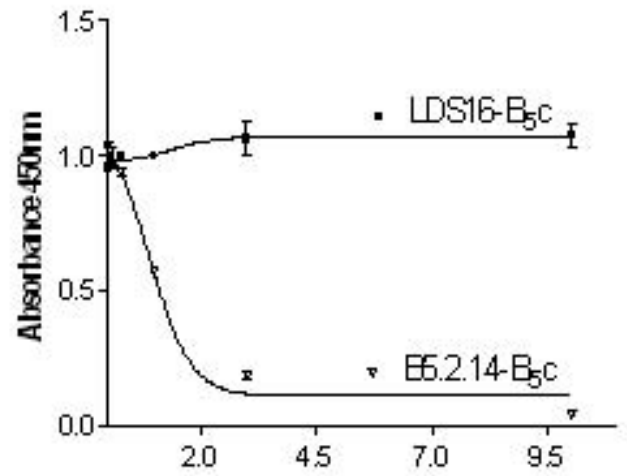

B1 (DTT) concentration per well - ugint

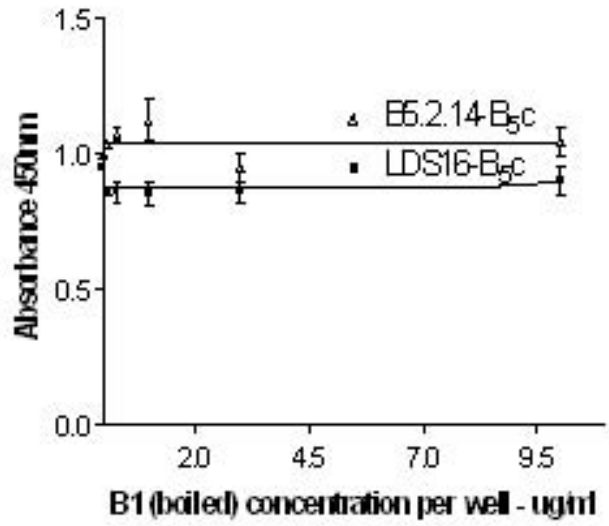

(D)

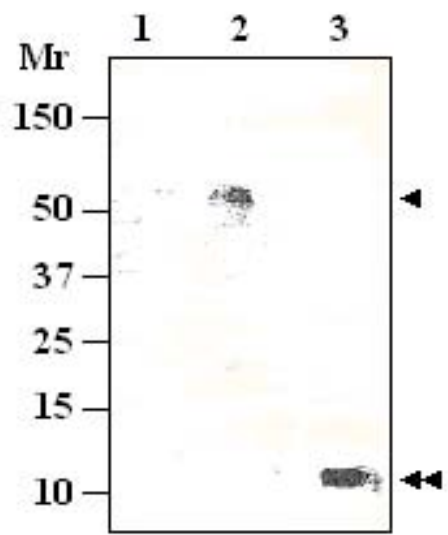


(A)

partially folded

folded EtxB monomer $\quad$ EtxB monomer

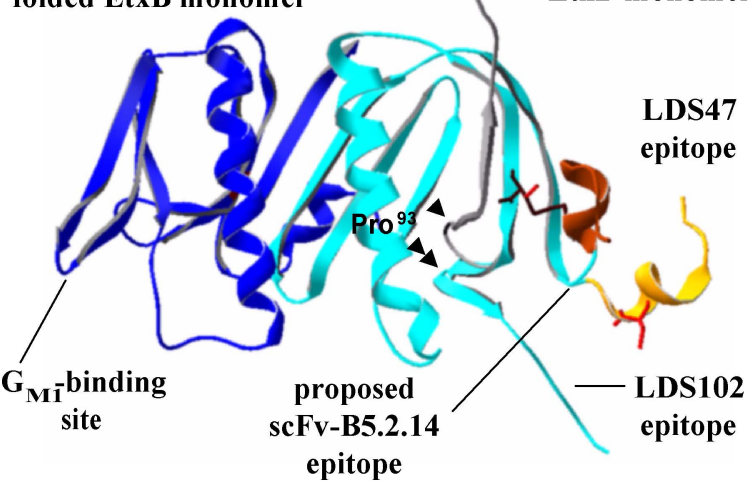

(B)

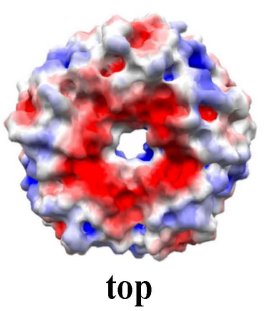

(C)

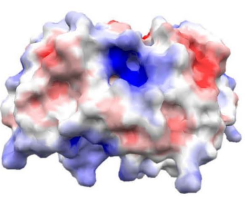

side

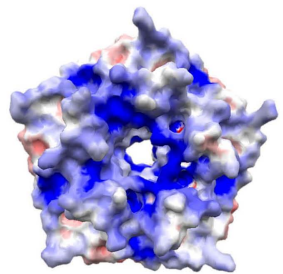

bottom

(D)

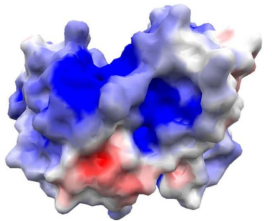

scFv-B5.2.14

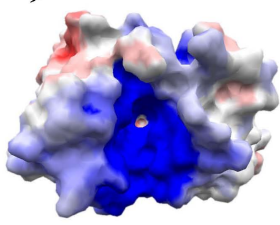

scFv-B1.3.9 